\title{
POTENCIALIDADE DE BIOMPHALARIA TENAGOPHILA DO LAGO DA PAMPULHA, BELO HORIZONTE, MG, COMO HOSPEDEIRA DO SCHISTOSOMA MANSONI
}

\author{
CECILIA PEREIRA DE SOUZA, NEUSA ARAÚJO, OMAR DOS SANTOS CARVALHO \\ \& JOSÉ RABELO DE FREITAS * \\ Centro de Pesquisas René Rachou - FIOCRUZ, Caixa Postal 1743, 30161 Belo Horizonte, MG, Brasil \\ * Departamento de Biologia, ICB, UFMG, Belo Horizon te, MG, Brasil
}

Potentiality of Biomphalaria tenagophila from Pampulha lake, Belo Horizonte, Minas Gerais State, as a host of Shistosoma mansoni - Biomphalaria tenagophila snails, from a population originally obtained from "Pampulha" lake, Belo Horizonte, Minas Gerais State, Brazil, were exposed to miracidia from four strains of Schistosoma mansoni: " $L E$ " and "HK" from Belo Horizonte, "AL" from Alagoas and "SJ" from $S \tilde{a}$ o Jose dos Campos, São Paulo. The "LE", "AL" and "SJ" strains are maintained in the laboratory and the " $H K$ " strain was obtained from feces of a patient residing near to "Pampulha" lake. Infection rates were of 4\% ("LE" strain), 6\% ("HK" strain), 30\% ("SJ" strain) and 40\% ("AL" strain). These infection rates were similar to those obtained by others authors for B. tenagophila from Minas Gerais. Experimentally infected snails when compared to $\mathrm{B}$. glabrata of the control group and $\mathrm{B}$. tenagophila naturally infected in "Pampulha" lake shed similar number of cercariae (2000 cercariae/snail). The high density of B. tenagophila in the "Pampulha" lake, the number of cercariae shed by naturally infected snails, the great number of persons who use the water for fishing and swimming, and the water contamination with human feces, are favourable factors for growing the Schistosomiasis focus in the lake.

Key words: Biomphalaria tenagophila - Schistosomiasis - Pampulha lake - Belo Horizonte

A distribuição geográfica dos moluscos hospedeiros intermediários do Schistosoma mansoni em Minas Gerais é bastante ampla (Paraense, 1972). A Biomphalaria glabrata é a espécie predominante, mas a $B$. straminea e a $B$. tenagophila são encontradas em diversas regiões do Estado. Alguns estudos têm demonstrado, entretando, que em certas áreas tem ocorrido substituição total ou parcial de B. glabrata por B. tenagophila (Paraense, 1970; Barbosa, 1973; Kawazoe et al., 1980). Segundo Carvalho et al., (1985a) este processo de exclusão estaria também em curso no lago da Pampulha. Além disso, essa espécie, que não era encontrada com infecção natural pelo S. mansoni, em Minas Gerais, passou, a partir de 1982 , a ser capturada albergando o parasito em questão (Melo et al., 1982; Carvalho et al., 1985a; Carvalho et al., 1985b).

A B. tenagophila foi encontrada pela primeira vez no lago da Pampulha em 1970 (Milward de Andrade, 1972), ocasião em que foram coletados 28 exemplares do planorbídeo. Daí para cá houve um aumento gradativo da densidade populacional da espécie, que atualmente se encontra dispersa por todo o perímetro do lago. De maio de 1981 a abril de 1984 foram coletados, naquela coleção hídrica, 3.695 exempla-

Trabalho parcialmente financiado pela SUDECAP, Belo Horizonte, MG.

Recebido em 10 de junho de 1986.

Aceito em 29 de dezembro de 1986. res de B. tenagophila e 467 de $B$. glabrata. No final de 1984 ocorreu ainda o primeiro encontro de $B$. tenagophila com infecção natural por $S$. mansoni no local (Carvalho et al., 1985a). As maiores densidades planorbídicas foram detectadas nas entradas dos córregos tributários na represa e em áreas próximas aos clubes Belo Horizonte, PIC e restaurante Redondo, no período de agosto a outubro de 1985.

Devido à alta densidade planorbídica atual em alguns pontos do lago da Pampulha, à utilização do mesmo para pesca e lazer, à presença, em pequena escala, de $B$. tenagophila com infecção natural, procedeu-se ao presente estudo com o objetivo de conhecer melhor a potencialidade desta população de molusco, como hospedeira intermediária do $S$. mansoni.

\section{MATERIAL E MÉTODOS}

Caramujos B. tenagophila, criados em laboratório, descendentes de exemplares coletados no lago da Pampulha, Belo Horizonte, MG, foram expostos a miracídios de quatro cepas de $S$. mansoni: LE e HK de origem local, Belo Horizonte (MG), AL de Alagoas e SJ de São José dos Campos (SP). As cepas LE, AL e SJ são mantidas em nosso laboratório, em B. glabrata, e HK foi obtida de fezes de paciente que reside nas margens do Ribeirão do Onça, efluente da Pampulha. As técnicas para obtenção de miracídios e infecção de moluscos foram descritas em trabalho anterior (Souza et al., 1979). 
TABELA I

Infecção experimentai de B. tenagophila do lago da Pampulha com quatro cepas de Schistosoma mansoni

\begin{tabular}{|c|c|c|c|c|c|c|c|}
\hline $\begin{array}{l}\mathrm{N}^{\mathrm{O}} \cdot \mathrm{de} \\
\text { moluscos } \\
\text { expostos }\end{array}$ & $\begin{array}{l}\text { Diâmetro } \\
(\mathrm{mm})\end{array}$ & $\begin{array}{c}\text { Cepa } \\
\text { de } \\
\text { S. mansoni }\end{array}$ & $\begin{array}{l}\text { Procedência } \\
\text { do } \\
\text { S. mansoni }\end{array}$ & $\begin{array}{c}\mathrm{N}^{\mathrm{O}} \mathrm{de} \\
\text { miracídios } \\
/ \text { moluscos }\end{array}$ & $\begin{array}{c}N^{\circ} \text { de } \\
\text { moluscos } \\
\text { sobreviventes }\end{array}$ & $\begin{array}{l}\mathrm{N}^{\mathrm{O}} \text { de } \\
\text { moluscos } \\
\text { positivos }\end{array}$ & $\begin{array}{c}\% \\
\text { de } \\
\text { infecção }\end{array}$ \\
\hline 50 & $5-12$ & LE & $\mathrm{MG}$ & 100 & 44 & 2 & 4,0 \\
\hline 50 & $4-10$ & $\mathrm{HK}^{* *}$ & MG & 100 & 42 & 3 & 6,0 \\
\hline 50 & $4-10$ & SJ & SP & 100 & 32 & 15 & 30,0 \\
\hline 50 & $4-10$ & $\mathrm{AL}$ & $\mathrm{AL}$ & 100 & 49 & 20 & 40,0 \\
\hline
\end{tabular}

(*) 50 dias após exposição a miracídios.

(**) Paciente residente à margem do Ribeirão do Onça, efluente da Pampulha.

Grupos de 50 exemplares com 4-12mm de diâmetro foram expostos em massa a 100 miracídios/molusco de cada uma das cepas do trematódeo. Concomitantemente, foram feitos controles de infecção com B. glabrata $(8-10 \mathrm{~mm})$ criada no laboratório, utilizando 20 miracídios de cada linhagem do trematódeo por molusco. Os moluscos foram examinados em microscópio estereoscópico após exposição à luz, com 40 dias e, após esmagamento, 50 dias depois da infecção, procedimento usado também para examinar os exemplares mortos durante o experimento. Os caramujos positivos do experimento $e$ do controle foram separados em aquários.

Paralelamente, foram coletados caramujos B. tenagophila no lago, para exame em laboratório e separaçāo de positivos. Cercárias obtidas de dois exemplares de $B$. tenagophila com infecção natural foram inoculadas em 10 camundongos, \pm 100 cercárias/animal. Os animais foram sacrificados 50 dias após a inoculação para coleta de ovos de $S$. mansoni e infecção de caramujos.

\section{RESULTADOS}

Os moluscos mostraram-se suscetíveis às quatro cepas de $S$. mansoni utilizadas. As taxas de infecçāo variaram de 4 a $40 \%$. Os resultados são mostrados na Tabela I. A B. tenagophila da Pampulha mostrou menor suscetibilidade a $S$. mansoni de cepas autóctones e maior às cepas alćctones. A mortalidade dos moluscos testados variou de 2 a $36 \%$. As taxas de infecção dos grupos de controle constituídos por B. glabrata foram de $48 \%$ (LE), $18 \%$ (HK), $22 \%(\mathrm{AL})$ e $24 \%(\mathrm{SJ})$ e a mortalidade variou de 42 a $76 \%$.

O número de cercárias eliminadas por exemplares infectados experimentalmente, após 90 minutos de exposição à luz, foi semelhante ao eliminado por $B$. glabrata do controle (cerca de 2.000 cercárias/molusco).
Dois exemplares de $B$. tenagophila capturadas no lago com infecção natural, no laboratório eliminaram em média 2.000 cercárias/molusco, após 90 minutos de exposição à luz.

Não foram obtidos ovos de $S$. mansoni do fígado nem do mesentério dos camundongos inoculados com cercárias de $B$. tenagophila infectada na Pampulha. Somente vermes machos, de corpo mais alongado que o normal, foram observados no mesentério.

\section{DISCUSSÃO}

A baixa suscetibilidade experimental de populaçōes de $B$. tenagophila de Minas Gerais a cepas de $S$. mansoni foi observada anteriormente por vários autores. A B. tenagophila procedente de diferentes regiões de Minas Gerais, de modo geral, tem apresentado maior afinidade à infecção com a cepa SJ de São José dos Campos (SP), mais adaptada a essa espécie de molusco (Paraense \& Corrêa, 1978, 1981; Santos et al., 1979; Carvalho, et al., 1979; Carvalho \& Souza, 1979, 1980; Souza et al., 1983; Souza, 1986).

Corrêa et al., (1979), testando a suscetibilidade experimental de B. tenagophila da Pampulha, Belo Horizonte, MG, às cepas SJ e LE, obtiveram taxas de infecção de 35 e $0 \%$, respectivamente, com as duas linhagens do trematódeo.

No presente estudo, os resultados foram semelhantes aos obtidos por Corrêa et al., (1979), em relação a SJ. As taxas de infecção experimental foram de $4 \%$ (LE), 6\% (HK), 30\% (SJ) e $40 \%$ (AL). Esses resultados já mostram, entretanto, maior grau de adaptação do molusco às cepas locais do parasito, apesar das taxas baixas de infecção.

O número de cercárias eliminadas por $B$. tenagophila infectada experimentalmente foi comparável ao de $B$. glabrata do controle, e ao de $B$. tenagophila, capturada no lago com infecção natural (2.000 cercárias/molusco). As 
cercárias provenientes de $B$. tenagophila capturada no logo, quando inoculadas em camundongos, deram origem somente a vermes machos.

Dados limnológicos obtidos por Freitas et al., (1986) mostram que as águas da represa apresentam um grau muito elevado de eutrofizaçāo, exibindo taxas de poluiçäo bastante acima daquelas permitidas para lagos frequentados pelo público em busca de recreaçāo ou pesca. Como a B. tenagophila é uma espécie em expansão no local, apresentando as maiores densidades nas áreas mais poluidas, entradas dos córregos, pode-se supor que sua resistência à poluição é maior do que a de $B$. glabrata, que é encontrada em baixa densidade também nos pontos de confluência dos córregos, de onde deve vir carreada pelas águas.

Do exposto, conclui-se que a $B$. tenagophila da Pampulha, apesar de apresentar taxas baixas de infecção natural e experimental (com cepas autóctones), deve ser olhada como um hospedeiro intermediário do $S$. mansoni em potencial, em condições favoráveis. De fato, nas condicōes atuais do lago, a alta densidade planorbidica em alguns pontos, o número de cercárias eliminadas por exemplares com infecção natural, o grande número de pessoas que entram em contato com as águas para pescar e nadar, a contaminação das águas por dejetos humanos, provenientes de esgotos demésticos, favorecem a inter-relação parasito hospedeiro invertebrado e vertebrado. A manutenção do ciclo do parasito no lago, então, poderá ser garantida pela presença do planorbídeo e do doente autóctone, portador de cepa de $S$. mansoni pré-adaptada ao molusco local (Magalhães et al., 1967).

Levando-se em consideração esse conjunto de fatores o lago da Pampulha corre o risco de voltar à sua antiga importância epidemiológica como foco de esquistossomose, se medidas profiláticas adequadas não forem tomadas a curto prazo.

\section{RESUMO}

Caramujos Biomphalaria tenagophila descendentes de exemplares coletados no lago da Pampulha, Belo Horizonte, Minas Gerais, foram expostos a miracidios de quatro cepas de Schistosoma mansoni: LE e HK de origem local, Belo Horizonte, AL do Estado de Alagoas e SJ, de São José dos Campos, SP. As cepas LE, AL c SJ são mantidas em laboratório e HK foi obtida de fezes de paciente que reside próximo à Pampulha.

As taxas de infecção experimental foram de $4 \%$ (LE), 6\% (HK), 30\% (SJ) e 40\% (AL). Esses indícios de infecção foram semelhantes aos obtidos por vários autores para populações de $B$. tenagophila de Minas Gerais. Caramujos infectados experimentalmente eliminaram número de cercárias comparável ao de $B$. glabrata do controle e de $B$. tenagophila capturada no lago, com infeccão natural (cerca de 2.000 cercárias) molusco). Devido à alta densidade planorbídica atual em alguns pontos do lago, número de cercárias eliminadas por exemplares naturalmente infectados, afluxo de pessoas para pesca e lazer, contaminação das águas por dejetos humanos, os autores alertam para o risco de crescimento do foco de esquistossomose no local.

Palavras-chave: Biomphalaria tenagophila -- esquistossomose - lago da Pampulha - Belo Horizonte

\section{AGRADECIMENTOS}

A Dilcia Maria Repetição, bolsista da FUNDEP, pela assistência técnica.

A Elzira Pereira da Silva, pelo serviço datilo. gráfico.

\section{REFERẼNCIAS}

BARBOSA, F. S., 1973. Possible competitive displacement and evidence of hybridization between two brasilian species of planorbid snails. Malacogia, 14: 401-408.

CARVALHO, O. S.; MILWARD DE ANDRADE, R. \& SOUZA, C. P., 1979. Susceptibilidade de Biomphalaria tenagophila (d'Orbigny, 1835) de Itajubá (MG), à infecção pela cepa "LE" de Schistosoma mansoni Sambon, 1907, de Belo Horizonte, MG (Brasil). Rev. Saúde Públ. São Paulo, 13: 2025.

CARVAlHO, O. S. \& SOUZA, C. P., 1979. Comportamento de Biomphalaria tenagophila (d'Orbigny, 1835) de Itajubá (MG, Brasil), exposta à cepa "SJ" de Schistosoma mansoni Sambon, 1907. Resumo IV Congresso Sociedade Brasileira de Parasitologia, Campinas, SP.

CARVAlHO, O. S. \& SOUZA, C. P., 1980. Suscetibilidade de Biomohalaria tenagophila (d'Orbigny, 1835 ) originária de Ravena, Município de Sabará, MG (Brasil), à cepa "LE" de Schistosoma mansoni. Anais V Congresso Brasileiro de Parasitologia, Rio de Janeiro, RJ

CARVALHO, O. S.; GUIMARÄES, C. T.; MASSARA, C. L. \& BONESIO, J. E. R., 1985a. Situação atual da esquistossomose mansoni no lago da Pampulha, Belo Horizonte, MG, Brasil. Rev. Saúde Pübl. Sāo Paulo, 19: 270-277.

CARVALHO, O. S.; SOUZA, C. P. \& KATZ, N., 1985b. Primeiro encontro de Biomphalaria tenagophila (d'Orbigny, 1835) naturalmente infectada com Schistosoma mansoni em Itajubá, sul do Estado de Minas Gerais, Brasil. Rev. Saúde Públ. São Paulo, 19: 88-91.

CORRËA, M. C. R.; COELHO, P. M. Z. \& FREITAS, J. R., 1979. Susceptibilidade de linhagens de Biom. phalaria tenagophila e Biomphalaria glabrata a duas cepas de Schistosoma mansoni (LE-Belo Horizonte e SJ-São José dos Campos). Rev. Inst. Med. Trop. Säo Paulo, 21: 72-76.

FREITAS, J. K.; GIANI, A. \& PINTO COELHO, R. M., 1986. Distribuição espacial e aspectos sazonais da comunidade planctônica na Represa da Pampulha. In: Relatório Final de Atividades, Convênio 
SUDECAP - Pampulha, Belo Horizonte, 1-72.

KAWAZOE, U.; MAGALHĀES, L. A.; HOTTA, L. K. \& TAKAKU, L., 1980. Competição biológica entre Biomphalaria glabrato (Say, 1818) e Biom phalaria tenagophila (D'Orbigny, 1835), em criadouros naturais no município de Ourinhos, SP (Brasil), Rev. Saúde Públ. São Paulo, 14: 65-87.

MAGALHĀES, L. A.; CAMARGO, L. A. P.; MUNIZ, J. R. O. \& ANDRADE, D., 1967. Novo foco de esquistossomose mansoni na cidade de Campinas (Estado de São Paulo, Brasil). Rev. Inst. Med. Trop. Sāo Paulo, 9: 378-380.

MELO, A. L.; PEREIRA, L. H. \& CORREA, M. C. R., 1982. Sobre o encontro de Biomphalaria tenagophila naturalmente infectada com Schistosoma mansoni no município de Jaboticatubas, Minas Gerais. VIII Congresso Brasileiro de Parasitologia. Resumos, p. 63.

MILWARD DE ANDRADE, R., 1972. Primeiro encontro de Biomphalaria tenagophila (d'Orbigny, 1835) no Lago da Pampulha, Belo Horizonte, MG. Ciência e Cultura, 24 (Supl.): 375.

PARAENSE, W. L., 1970. Planorbídeos hospedeiros intermediários do Schistosoma mansoni. In: $E_{S}$ quistossomose mansoni. CUNHA, A. S., Sarvier \& Ed. Univ. São Paulo, Cap. 2, 13-30.

PARAENSE, W. L., 1972. Fauna planorbídica do Brasil. In: Introducão à Geografia Médica do Brasil. LACAZ, C. S.; BARUZZI, G. R. \& SIQUEIRA, Jr. W. Eds. São Paulo, Edgard Blïcher, Ed. Univ.
Săo Paulo, Cap. 10, 213-239.

PARALNSE, W. L. \& CORREA, L. R. 1978. Differential susceptibility of Biomphalaria tenagophila populations to infection with a strain of Schistosoma mansoni. J. Parasitol. 64: 822-826.

PARAENSE, W. L. \& CORREA, L. R. 1981. Observations on two biological races of Schistosoma mansoni. Mem. Inst. Oswaldo Cruz, 76: 287-291.

SANTOS, M. B. L.; FREITAS, J. R.; CORREA, M. C. R. \& COELHO, P, M. Z., 1979. Suscetibilidade ao Schistosoma mansoni de híbridos de Biomphalaria tenagophila do Taim, RS, Cabo Frio, RJ e Belo Horizonte-MG. Rev. Inst. Med. Trop. São Paulo, 21: 281-286.

SOUZA, C. P., 1986. Estudo de moluscos do gênero Biomphalario de Minas Gerais, com relação a adaptação parasito-hospedeiro e importância na epidemiologia da esquistossomose. Rev. Inst. Med. Trop. Sāo Paulo, 28: 287-292.

SOUZA, C. P.; ARAUJO, N.; MADEIRA, N. G. \& CARVALHO, O. S. 1983. Suscetibilidade de Biomphalaria tenagophila de Belo Horizonte e adjacências à infecção com três cepas de Schistosoma mansoni. Rev. Inst. Med. Trop. São Paulo, 25: 168-172.

SOUZA, C. P.; DIAS, E. P.; AZEVEDO, M. L. L. \& PAULINI, E., 1979. Observações sobre alguns fa. tores que influem na manutençāo do Schistosoma mansoni em laboratório. Rev. Brasil. Pesq. Med. Biol., 12: 411-419. 